\title{
Noninvasive Evaluation of Injectable Chitosan/Nano-Hydroxyapatite/Collagen Scaffold via Ultrasound
}

\author{
Yan Chen,, ${ }^{1}$ Songjian Li, ${ }^{2}$ Xiaoming Li, ${ }^{3}$ Yichen Zhang, ${ }^{2}$ Zhi Huang, ${ }^{4}$ Qingling Feng, ${ }^{5}$ \\ Zhilai Zhou, ${ }^{2}$ Bomiao Lin, ${ }^{6}$ and Bo Yu$^{2}$ \\ ${ }^{1}$ Department of Ultrasonic Diagnosis, Zhujiang Hospital of Southern Medical University, Guangzhou 510282, China \\ ${ }^{2}$ Department of Orthopedics, Zhujiang Hospital of Southern Medical University, Guangzhou 510282, China \\ ${ }^{3}$ Key Laboratory for Biomechanics and Mechanobiology of Ministry of Education, School of Biological Science and Medical Engineering, \\ Beihang University, Beijing 100191, China \\ ${ }^{4}$ School of Geosciences and Info-Physics, Central South University, Changsha 410083, China \\ ${ }^{5}$ State Key Laboratory of New Ceramic and Fine Processing, Tsinghua University, Beijing 100084, China \\ ${ }^{6}$ Department of Radiology, Zhujiang Hospital of Southern Medical University, Guangzhou 510282, China
}

Correspondence should be addressed to Xiaoming Li, x.m.li@hotmail.com and Bo Yu, gzyubo@gmail.com

Received 1 March 2012; Accepted 3 April 2012

Academic Editor: Shuming Zhang

Copyright ( $) 2012$ Yan Chen et al. This is an open access article distributed under the Creative Commons Attribution License, which permits unrestricted use, distribution, and reproduction in any medium, provided the original work is properly cited.

\begin{abstract}
To meet the challenges of designing an in situ forming scaffold and regenerating bone with complex three-dimensional (3D) structures, an in situ forming hydrogel scaffold based on nano-hydroxyapatite (nHA), collagen (Col), and chitosan (CS) was synthesized. Currently, only a limited number of techniques are available to mediate and visualize the injection process of the injectable biomaterials directly and noninvasively. In this study, the potential of ultrasound for the quantitative in vivo evaluation of tissue development in CS/nHAC scaffold was evaluated. The CS/nHAC scaffold was injected into rat subcutaneous tissue and evaluated for 28 days. Quantitative measurements of the gray-scale value, volume, and blood flow of the scaffold were evaluated using diagnostic technique. This study demonstrates that ultrasound can be used to noninvasively and nondestructively monitor and evaluate the in vivo characteristics of injectable bone scaffold. In comparison to the CS, the CS/nHAC scaffold showed a greater stiffness, less degradation rate, and better blood supply in the in vivo evaluation. In conclusion, the diagnostic ultrasound method is a good tool to evaluate the in vivo formation of injectable bone scaffolds and facilitates the broad use to monitor tissue development and remodeling in bone tissue engineering.
\end{abstract}

\section{Introduction}

Each year, more than a million people worldwide require bone grafts to create or repair bone. Currently, autografts are the gold-standard treatment to replace or augment bone. However, because autografts must be taken from a separate donor site on the patient's body and, therefore, require two surgical procedures, there is a limited supply of available bone as well as serious risk of donor-site pain, infection, and bleeding. To overcome these issues, there is a significant clinical need to develop alternatives to autografts and allografts for bone grafting procedures. From a clinical perspective, the use of injectable scaffolds is very attractive as it minimizes patient discomfort, risk of infection, scar formation, and the cost of treatment [1].
These scaffolds are liquid before and during the injection, but they undergo gelation in situ, during the procedure of surgery, allowing them to fill in a bone defect of any shape. Chitosan is currently seen as a promising biodegradable biomaterial, while $\beta$-glycerophosphate (GP) is a well-known biocompatible agent [2-4]. The chitosan solution with GP salts possesses a physiological $\mathrm{pH}$ and can be held liquid below room temperature for encapsulating living cells and therapeutic proteins [5-7]. Recent studies have demonstrated the application of the C/GP hydrogel as an injectable scaffold for tissue engineering [8-11].

Bone is mainly composed of nano-hydroxyapatite and collagen fibers, in which the $c$-axes of the HA are regularly aligned along the collagen fibers [12-14]. While considerable effort has gone into determining the relationship between 
collagen structure and mineral orientation, synthetic recreation of this most fundamental level of bone structure has eluded the materials engineer seeking to fabricate bonelike composites. It would be desirable to mimic both the composition and structure of bone for synthetic bone graft substitutes [15-17]. Biomimetic bone materials can be used in conjunction with natural bone, to induce new bone tissue formation and promote bone remodeling. At present this is the most promising route for the repair of defects in natural bone [18-22]. The ideal bone scaffold should promote early mineralization and support new bone formation while at the same time allowing for replacement by new bone. A bone-like nano-hydroxyapatite/collagen (nHAC) composite by mineralizing type 1 collagen seems to be a very promising system for bone reconstructive or regenerative surgery. In our preliminary study, a new type of thermosensitive material based on CS, HA, and Col was fabricated with a biomimetic strategy. NHAC uniformly dispersed in the chitosan matrix and this CS/nHAC composite showed some features of natural bone. Thus, the CS/nHAC scaffolds have shown to possess promising physicochemical and biological properties for use in bone tissue engineering approaches [2326].

In the field of tissue engineering, there is a growing need for quantitative methods to analyze in situ and in real time the tissue development in three-dimensional scaffolds. Ultrasound is a well-known noninvasive and nondestructive imaging method [27-30]. The goal of the current study was to demonstrate the utility of ultrasound as a noninvasive, nondestructive tool to evaluate the in vivo development of the CS/nHAC scaffold under subcutaneous implantation on the back of rats for the period of 4 weeks.

\section{Materials and Methods}

2.1. Materials. Medical grade type 1 collagen powder was purchased from YierKang Company (China), medical grade chitosan (Mw 250000 and a degree of deacetylation of 95.6\%) was provided by Shandong AKBiotech Ltd (China), and $\beta$-glycerophosphate $\left(\mathrm{GP}, \mathrm{C}_{3} \mathrm{H}_{7} \mathrm{Na}_{2} \mathrm{O}_{6} \mathrm{P}\right)$ was from Sigma (USA). The water used in the experiments was triply distilled.

2.2. Preparation of CS/nHAC and CS. The CS/nHAC composite was prepared by the procedure reported previously. Firstly, nHAC powder was synthesized by self-assembly of nanofibrils of mineralized collagen and sterilized by $\gamma$ ray irradiation $(1.5 \mathrm{Mrad})$. Secondly, chitosan $(2 \mathrm{~g})$ was dissolved in hydrochloric acid solution $(98 \mathrm{~mL}, 0.1 \mathrm{M}$ ). Thirdly, the nHAC powder was added to the chitosan solution $(0.02 \mathrm{~g} / \mathrm{mL})$ with the ratio of $1: 1$ in weight. Finally, the $\mathrm{pH}$ of the CS/nHAC solution was adjusted to 7.0 by adding droplets of $\beta$-glycerophosphate solution ( $30 \%(\mathrm{w} / \mathrm{v})$ ).

The CS was prepared similar to the procedure of $\mathrm{CS} / \mathrm{nHAC}$. Chitosan (2 $\mathrm{g}$ ) was dissolved in hydrochloric acid solution $(98 \mathrm{~mL}, 0.1 \mathrm{M})$, and then the $\mathrm{pH}$ of the CS solution was adjusted to 7.0 by adding droplets of $\beta$-glycerophosphate solution $[30 \%(\mathrm{w} / \mathrm{v})]$. The samples for biological tests were prepared in line with the aseptic technique in an aseptic manipulation cabinet.

2.3. Implantation Experiments in Rats. All the animals were operated according to the guidelines for animal experiments. In this study, ten female Wistar rats (average body weight $300 \mathrm{~g}$ ) were carried out under general gas anesthesia strictly following a protocol approved by the Southern medical University Institutional Animal Care and Use Committee. Anesthesia consisted of $1 \%$ isoflurane delivered via facemask. The solutions were prepared by gently mixing $1 \mathrm{~mL}$ of CS and nHAC. A $1 \mathrm{~mL}$ syringe with a 26-gauge needle was used to inject the solution into the subcutaneous dorsum of a rat.

2.4. Ultrasound Image. The implants were imaged using an ALOKA prosound $\alpha-10$ premier diagnostic ultrasound system with a $12 \mathrm{MHz}$ transducer using the following parameters: a dynamic range of 55, a mechanical index of 1.1, a gain of 80 , and a depth of $3 \mathrm{~cm}$. Images were taken at $1 \mathrm{~h}$, 14 days, and 28 days after injection. Anesthetized rats were laid in the supine position, and an ultrasound transducer was positioned on the surface of the back. Images were taken always with the same settings (contrast: $50 \%$, brightness $40 \%$, and zoom: $100 \%$ ) at different time point. At each time point, five images from the scaffolds in each group were stored. The images were transferred to an offline computer (Q-lab, Philips Medical Systems) to measure the gray-scale value (GV) intensity. A region of interest (ROI) was set to encompass the entire scaffold. Next, the ultrasound system was set to color Doppler to detect the blood flow surround the scaffold.

2.5. Image Analysis. Ultrasound images are the visualization of the backscattered signal that arises due to the difference in mechanical impedance between different materials and phases. The backscattered signal is displayed as a gray-scale array with values ranging from 0 to 256 , with 0 indicating a negligible difference in impedance from the surrounding media; the development of an ultrasound signal over time was interpreted as an increase in stiffness that may due to the solidify development of the scaffold. The gray-scale value $(\mathrm{GV})$ was analyzed by measuring the mean GV of the implants over time by the method of histogram echo intensity. The measurements from the five images were averaged together for each implant. Implant volume was determined in vivo from the images of the total area of the scaffold. Since the images were binary, the number of implant pixels could be determined by summation of the pixels. All image analysis was performed using MATLAB.

2.6. Statistical Analysis. Statistical significance of differences was determined using one-way analysis of variance ANOVA. If differences were detected, pairwise comparisons were made using Turkey's HSD test at a confidence interval of 95\% $(P<$ 0.05). 


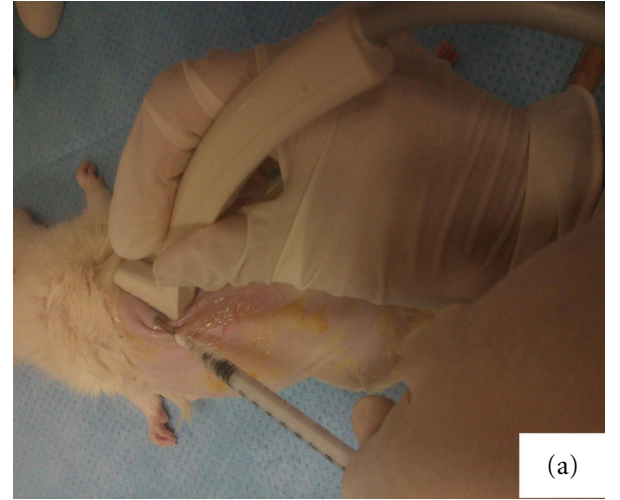

(a)

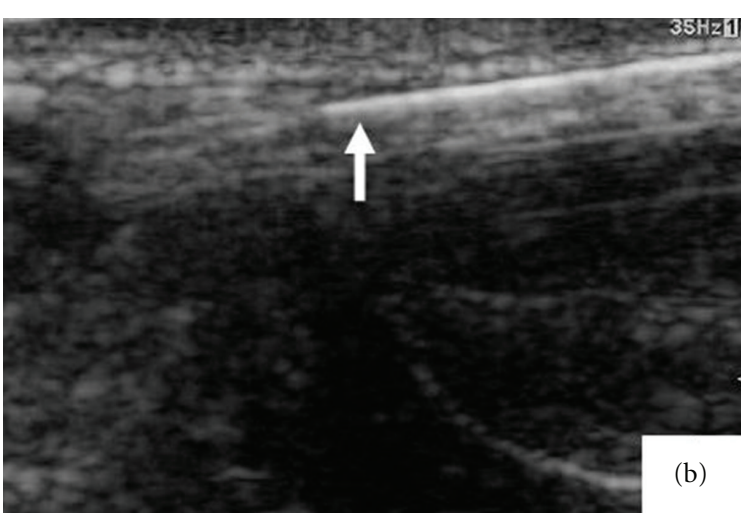

(b)

FIGURE 1: Ultrasound imaging of the injection process of the in situ formation of the CS and CS/nHAC gel: (a) subcutaneous injection of the CS/nHAC solution under the ultrasound transducer. (b) The injection process was monitored by ultrasound (arrow: the needle of the syringe).

\section{Result and Discussion}

Over the past decades, nano-biomaterials have played significant roles in the field of biomedical engineering and pharmaceutics. For successful clinical applications, the investigations into biocompatibility and toxicity of nano-biomaterials are research interests of great significances. The use of diagnostic ultrasound to study the in vivo compatibility of injectable nano-biomaterials is a novel means in a noninvasive, nondestructive manner. The primary advantage of this technique is the real-time visualization of the implant formation process. Long-term information regarding formation and degeneration behavior for the same implant over time can be obtained. In the current study, the utility of monitoring implant formation using ultrasound was demonstrated by analyzing the formation and degeneration of injectable chitosan and chitosan/nano-hydroxyapatite/collagen scaffold. Figure 1 showed the feasibility of using the ultrasound to manipulate the CS/nHAC system injecting into the Wistar back as in situ forming scaffold. The CS/nHAC solution was injected into the subcutaneous dorsum of the Wistar rat with a $1 \mathrm{~mL}$ syringe under the ultrasound transducer (Figure 1(a)); the needle and the process of the injection could be easily visualized by the ultrasound (Figure 1(b)).

3.1. Implant Formation. Ultrasound has been used to evaluate tissue properties of articular cartilage with the aim of developing a noninvasive clinical method to assess articular cartilage degeneration and repair [27-29]. Recently, it was also used to evaluate the in vivo tissue engineering structures. Kreitz et al. [30] found that the gray-scale value of the ultrasound demonstrated a good correlation with the hydroxyapatite content as marker of collagen formation and with the histological findings. Oe et al. [31] used the ultrasound to evaluate the cell numbers in bone marrow stromal cell/ $\beta$-tricalcium phosphate composites and found ultrasound effectively responded to the quantity of BMSC/ $\beta$ TCP composites and was well correlated to the actual number of cells contained. Therefore, we chose to use a
TABLE 1: The grey-scale value of ultrasound image on experimental group and control group after 0,14 , and 28 days of incubation. The values are shown as mean \pm standard deviation.

\begin{tabular}{lcccc}
\hline Group & Time (day) & $n$ & GV & $P$ value \\
\hline CS & 0 & 5 & $52.7 \pm 13.0$ & 1.00 \\
CS/nHAC & 0 & 5 & $51.4 \pm 11.5$ & \\
CS & 14 & 5 & $68.6 \pm 6.5$ & 0.80 \\
CS/nHAC & 14 & 5 & $81.1 \pm 4.6$ & \\
CS & 28 & 5 & $74.9 \pm 7.5$ & 0.02 \\
CS/nHAC & 28 & 5 & $99.8 \pm 33.2$ & \\
\hline
\end{tabular}

medical diagnostic ultrasound device with the advantage of ensuring the absence of damage to the surrounding tissues. Ultrasound images of the CS and CS/nHAC scaffold taken at different time points (days 0,14 , and 28) are shown in Figure 2. The average GV was intended to be used as an index to determine the degree of the hydroxyproline content as marker of collagen formation and implant stiffness, with lower mean GVs corresponding to a more fluid matrix and larger mean GVs correlating to solid implant. The ultrasound grey-scale signal increased with time, which may demonstrate production of extracellular matrix. The $\mathrm{CS} / \mathrm{nHAC}$ had a mean GV of $51.7 \pm 11$ after $1 \mathrm{~h}$ of injection; the mean GV increased to $81.1 \pm 4.6$ after 14 days and attained the highest mean GV of $107.8 \pm 22$ after 28 days. Significant differences were observed between the GVs on day 1, 14, and 28 in CS/nHAC group. In contrast, the CS scaffold had a mean GV of $52.7 \pm 13$ after $1 \mathrm{~h}$ of injection; the mean GV increased to $68.5 \pm 6.4$ after 14 days and had a maximum GV of $74.8 \pm 7.4$ after 28 days. But no significant differences were observed between the GVs on day 1, 14, and 28 in CS group. On day 28, the mean GVs of the CS/nHAC group were significantly higher than the CS group $(P<0.05)$ (Figure 3 , Table 1). Using this measurement, it was observed that the $\mathrm{CS} / \mathrm{nHAC}$ scaffold attained the highest gray-scale value at day 28 , indicating the CS/nHAC scaffold had the highest implant stiffness, which maybe related to the early osteogenesis. 


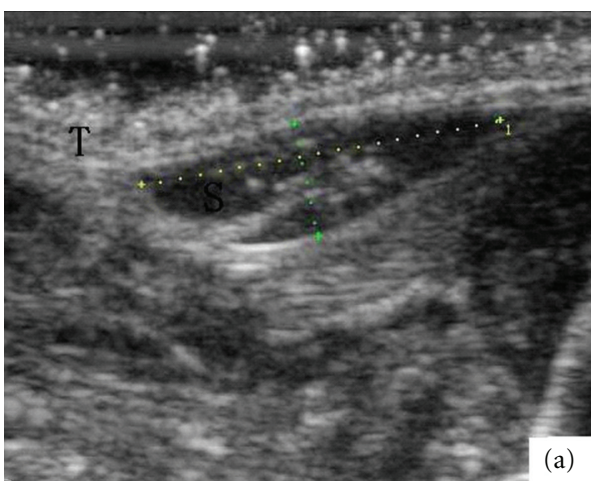

(a)

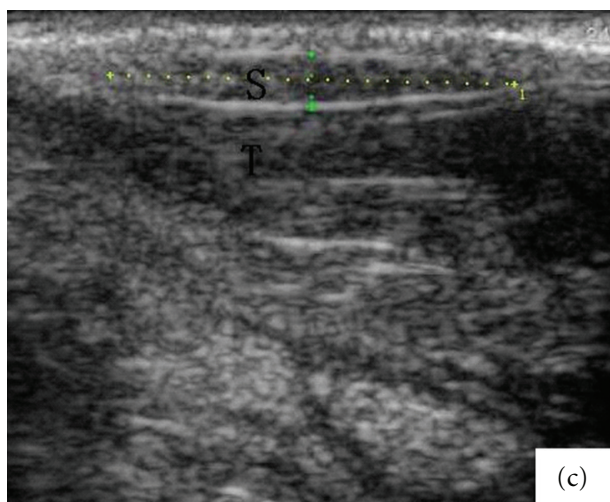

(c)

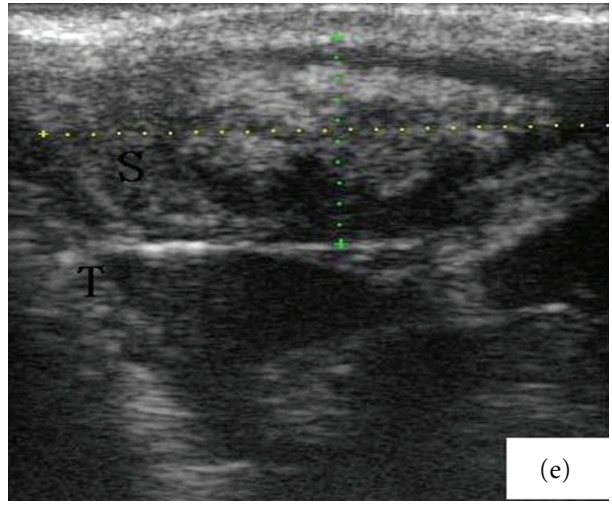

(e)

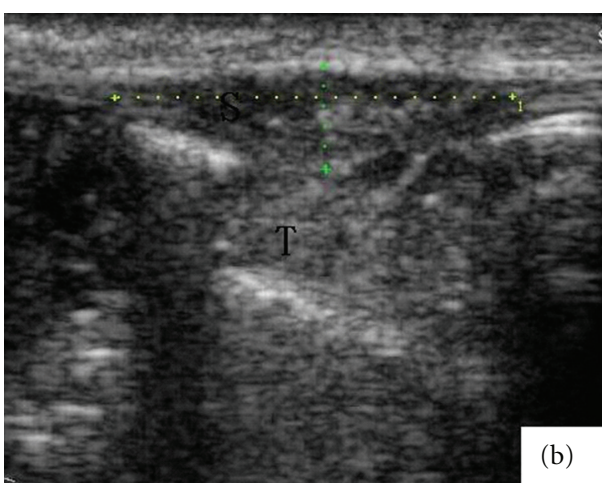

(b)

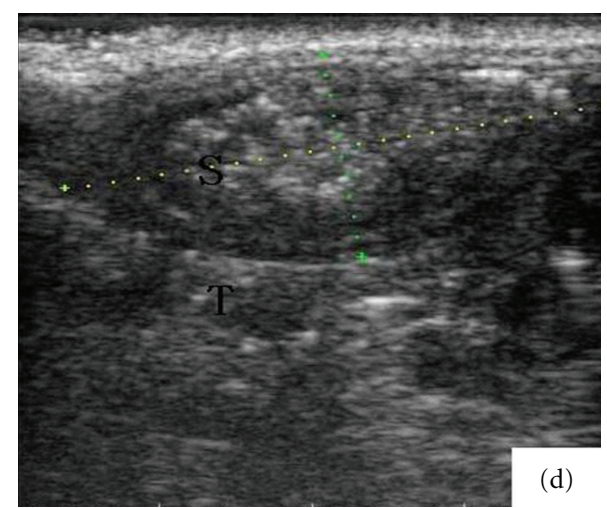

(d)

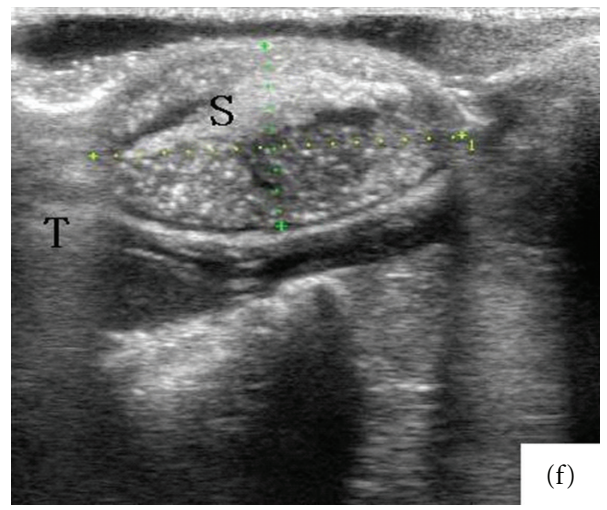

(f)

FIGURE 2: The ultrasound images of the scaffold taken on day 0 (a) and (d), day 14 (b) and (e), and day 28 (c) and (f). (CS scaffolds: (a), (b), (c); CS/nHAC scaffolds: (d), (e), (f); S: scaffold; T: surrounding tissues, cross-section area: the blue and yellow dot cross in the scaffold).

3.2. Implant Volume. The volumetric changes of the CS/nHAC and CS scaffold were assessed after $1 \mathrm{~h}, 14$ days, and 28 days, respectively. The use of percutaneous ultrasound would allow volume monitoring over time after administration of CS and CS/nHAC gel. This procedure of calculation has been used in radiology ultrasound for more than 20 years to measure breast cysts, renal cysts, and more. Volume calculation is reported in the literature that the detection volume may have 10 percent differences in two-dimensional ultrasound, which is at least as good as that obtained by three-dimensional calculation [32-34]. Implant degeneration was measured as a change in crosssectional area over time in both groups. The CS scaffold exhibited decreasing in size more than $80 \%$ over the 28 days. However, the CS/nHAC scaffold showed a slight decrease and remained $62 \%$ for the duration of 28 days (Table 2, Figure 4). Significant bigger cross-section area was observed in the CS/nHAC scaffold on day 14 (Figures 2(b) and 2(e), $P<0.05$ ) and day 28 (Figures $2(\mathrm{c})$ and $2(\mathrm{f}) P<0.01$ ). The results demonstrated a stable material degeneration rate of the CS/nHAC scaffold, which is suitable to be used as a bone substitute. However, the CS scaffold seemed to degenerate too fast in the first 4 weeks to be a good bone substitute.

3.3. Implant Blood Supply. The successful clinical outcome of the implanted tissue-engineered bone is dependent on 


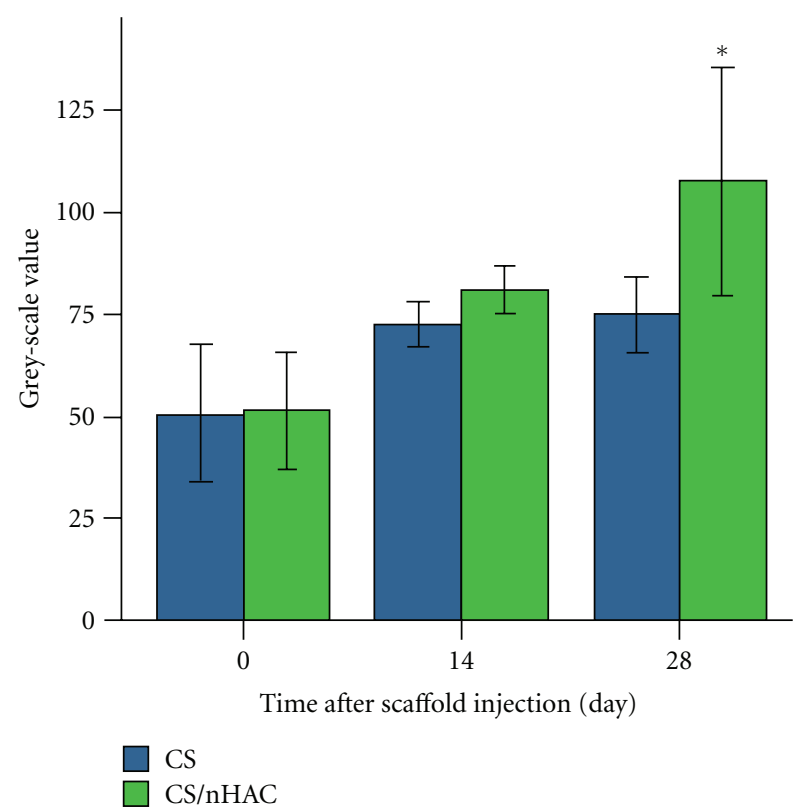

Figure 3: The grey-scale value of ultrasound image on experimental group and control group after 0,14 , and 28 days of incubation. The values are shown as mean \pm standard deviation $(n=5, * P<0.05)$.

TABLE 2: The cross-section area of ultrasound image on experimental group and control group after 0,14 , and 28 days of incubation. The values are shown as mean \pm standard deviation.

\begin{tabular}{lcccc}
\hline Group & Time (day) & $n$ & Pixel & $P$ value \\
\hline CS & 0 & 5 & $28982.8 \pm 6782.9$ & 0.16 \\
CS/nHAC & 0 & 5 & $35662.0 \pm 3321.5$ & \\
CS & 14 & 5 & $17084.8 \pm 256.3$ & 0.04 \\
CS/nHAC & 14 & 5 & $35662.0 \pm 6341.7$ & \\
CS & 28 & 5 & $5169.2 \pm 613.4$ & 0.00 \\
CS/nHAC & 28 & 5 & $22050.2 \pm 3449.4$ & \\
\hline
\end{tabular}

the establishment of a functional vascular network. So it is very important to evaluate the vascularization of the implant in vivo. The blood flow surrounding the implant was shown in Figure 5. In the CS scaffold group, no blood flow was detected by the color Doppler imaging during 28 days after implantation. However sporadic blood flow was found around the CS/nHAC scaffold on day 14 and 28 after implantation (Figure 5) which maybe related to the material vascularization. Although no blood flow was found inside the scaffold during the 28-day observation, the blood flow surrounding the materials indicates that there were new vessels surrounding the scaffold and new capillary may penetrate into the implant and form a network to promote osteogenesis. The use of ultrasound imaging is of growing interest as new technologies become available for in vivo analysis of blood flow. Despite some limitations including a lower degree of sensitivity to changes in tissue perfusion and the inability to assess rapid or transient changes in tissue perfusion, we found that the ultrasound method can be used successfully to examine regional blood perfusion of the biomaterials in vivo [35]. As this method does not involve a surgical procedure, ultrasound offers a rapid, noninvasive

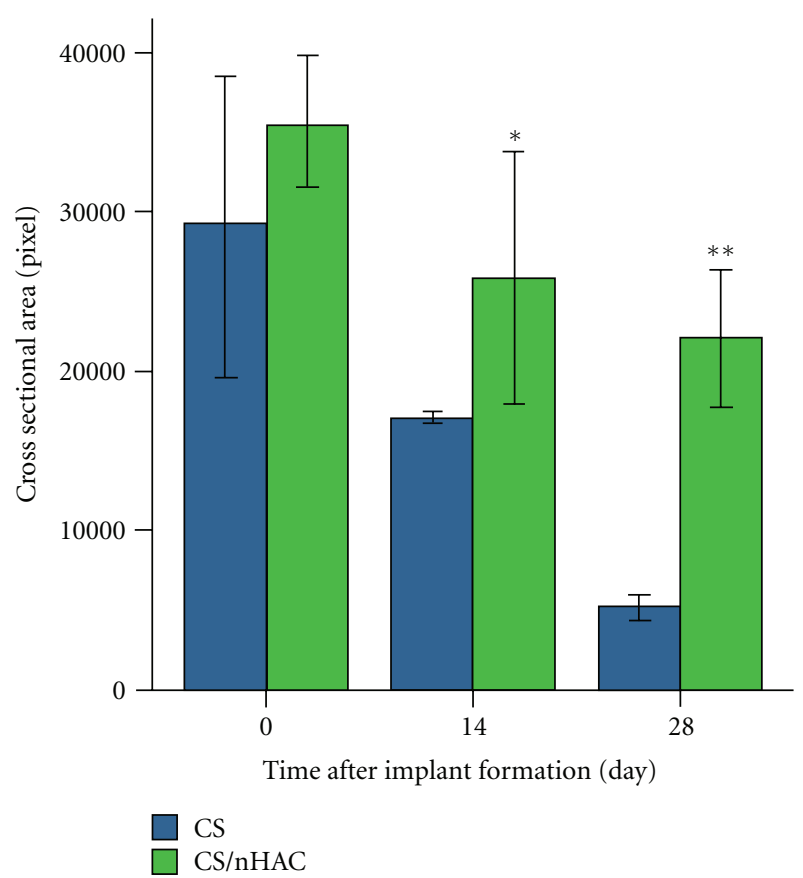

FIgURE 4: The cross-section area of ultrasound image on experimental group and control group after 0,14 , and 28 days of incubation. The values are shown as mean \pm standard deviation $\left(n=5,{ }^{* *} P<0.01,{ }^{*} P<0.05\right)$.

way to obtain hemodynamic measurements with little risk to the experimental subject.

\section{Conclusions}

This study has demonstrated that it is possible to noninvasively and nondestructively evaluate the volume, stiffness, and blood supply of the injectable bone scaffold using a diagnostic ultrasound, providing a new means for the biocompatibility study of in situ forming implants in in vivo applications. The primary advantage of this technique is the real-time visualization of the implant formation process. Through the use of quantitative image analysis techniques, long-term information regarding formation and degeneration behavior for the same implant over time can be obtained. In comparison to the CS, the CS/nHAC scaffold showed a greater stiffness, less degeneration rate, and better blood supply in the in vivo biocompatibility evaluation. The results shown in this paper support the proof of principle of using ultrasound for the evaluation of tissue development in hydrogel-based tissue-engineered structures.

\section{Authors' Contribution}

Y. Chen and S. Li contributed equally to this paper.

\section{Acknowledgments}

The authors are grateful for the financial support from National Natural Science Foundation of China (no. 81101348 and no. 31000431), Natural Science Foundation 


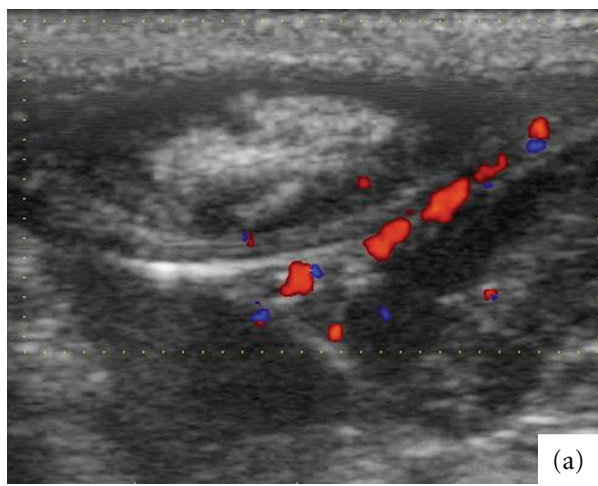

(a)

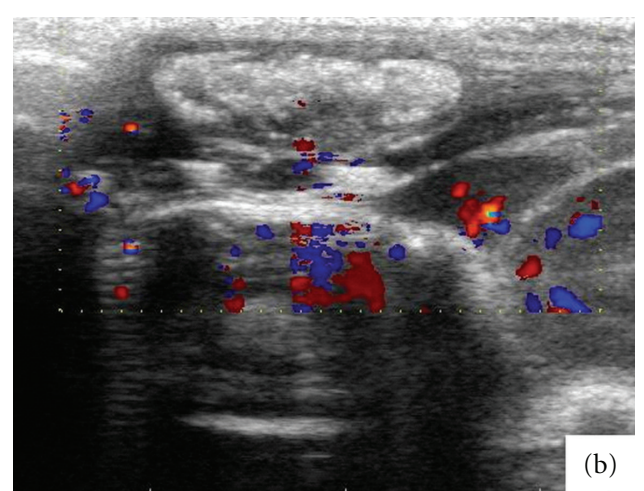

(b)

Figure 5: The blood supply to the CS/nHAC scaffold after 14 (a) and 28 days (b).

of Guangdong Province, China (10451051501004727), Guangdong Province Science and Technology project, China (ultrasound evaluation of injectable nanobiomaterials), Doctoral Fund of Ministry of Education, China (20114433120004), the Beijing Nova Program (no. 2010B011), and State Key Laboratory of New Ceramic and Fine Processing Tsinghua University, outstanding person fund of Zhujiang Hospital.

\section{References}

[1] Q. Hou, P. A. De Bank, and K. M. Shakesheff, "Injectable scaffolds for tissue regeneration," Journal of Materials Chemistry, vol. 14, no. 13, pp. 1915-1923, 2004.

[2] A. Chenite, M. Buschmann, D. Wang, C. Chaput, and N. Kandani, "Rheological characterisation of thermogelling chitosan/glycerol-phosphate solutions," Carbohydrate Polymers, vol. 46, no. 1, pp. 39-47, 2001.

[3] A. Chenite, C. Chaput, D. Wang et al., "Novel injectable neutral solutions of chitosan form biodegradable gels in situ," Biomaterials, vol. 21, no. 21, pp. 2155-2161, 2000.

[4] A. Chenite, S. Gori, M. Shive, E. Desrosiers, and M. D. Buschmann, "Monolithic gelation of chitosan solutions via enzymatic hydrolysis of urea," Carbohydrate Polymers, vol. 64, no. 3, pp. 419-424, 2006.

[5] E. Ruel-Gariépy, A. Chenite, C. Chaput, S. Guirguis, and J. C. Leroux, "Characterization of thermosensitive chitosan gels for the sustained delivery of drugs," International Journal of Pharmaceutics, vol. 203, no. 1-2, pp. 89-98, 2000.

[6] E. Ruel-Gariépy, G. Leclair, P. Hildgen, A. Gupta, and J. C. Leroux, "Thermosensitive chitosan-based hydrogel containing liposomes for the delivery of hydrophilic molecules," Journal of Controlled Release, vol. 82, no. 2-3, pp. 373-383, 2002.

[7] E. Ruel-Gariépy and J. C. Leroux, "Chitosan: a natural polycation with multiple applications," ACS Symposium Series, vol. 934, pp. 243-259, 2006.

[8] S. M. Richardson, N. Hughes, J. A. Hunt, A. J. Freemont, and J. A. Hoyland, "Human mesenchymal stem cell differentiation to NP-like cells in chitosan-glycerophosphate hydrogels," Biomaterials, vol. 29, no. 1, pp. 85-93, 2008.

[9] M. H. Cho, K. S. Kim, H. H. Ahn et al., "Chitosan gel as an in situ-forming scaffold for rat bone marrow mesenchymal stem cells in vivo," Tissue Engineering, Part A, vol. 14, no. 6, pp. 1099-1108, 2008.
[10] C. D. Hoemann, A. Chenite, J. Sun et al., "Cytocompatible gel formation of chitosan-glycerol phosphate solutions supplemented with hydroxyl ethyl cellulose is due to the presence of glyoxal," Journal of Biomedical Materials Research, vol. 83, no. 2, pp. 521-529, 2007.

[11] C. D. Hoemann, J. Sun, A. Légaré, M. D. McKee, and M. D. Buschmann, "Tissue engineering of cartilage using an injectable and adhesive chitosan-based cell-delivery vehicle," Osteoarthritis and Cartilage, vol. 13, no. 4, pp. 318-329, 2005.

[12] X. Li, C. A. van Blitterswijk, Q. Feng, F. Cui, and F. Watari, "The effect of calcium phosphate microstructure on bonerelated cells in vitro," Biomaterials, vol. 29, no. 23, pp. 33063316, 2008.

[13] X. Li, H. Liu, X. Niu et al., "Osteogenic differentiation of human adipose-derived stem cells induced by osteoinductive calcium phosphate ceramics," Journal of Biomedical Materials Research, vol. 97, no. 1, pp. 10-19, 2011.

[14] X. M. Li, X. H. Liu, M. Uo, Q. L. Feng, F. Z. Cui, and F. Watari, "Investigation on the mechanism of the osteoinduction for calcium phosphate," Bone, vol. 43, pp. 111-112, 2008.

[15] X. Li, Q. Feng, X. Liu, W. Dong, and F. Cui, "Collagen-based implants reinforced by chitin fibres in a goat shank bone defect model," Biomaterials, vol. 27, no. 9, pp. 1917-1923, 2006.

[16] X. Li, H. Gao, M. Uo et al., "Effect of carbon nanotubes on cellular functions in vitro," Journal of Biomedical Materials Research, vol. 91, no. 1, pp. 132-139, 2009.

[17] X. Li, H. Gao, M. Uo et al., "Maturation of osteoblast-like $\mathrm{SaoS}_{2}$ induced by carbon nanotubes," Biomedical Materials, vol. 4, no. 1, Article ID 015005, 2009.

[18] X. Li, Y. Fan, and F. Watari, "Current investigations into carbon nanotubes for biomedical application," Biomedical Materials, vol. 5, no. 2, Article ID 022001, 2010.

[19] X. Li, X. Liu, J. Huang, Y. Fan, and F. Z. Cui, "Biomedical investigation of CNT based coatings," Surface and Coatings Technology, vol. 206, no. 4, pp. 759-766, 2011.

[20] X. Li, Q. Feng, and F. Cui, "In vitro degradation of porous nano-hydroxyapatite/collagen/PLLA scaffold reinforced by chitin fibres," Materials Science and Engineering C, vol. 26, no. 4, pp. 716-720, 2006.

[21] X. M. Li, X. H. Liu, G. P. Zhang et al., "Repairing $25 \mathrm{~mm}$ bone defect using fibres reinforced scaffolds as well as autograft bone," Bone, vol. 43, pp. 94-94, 2008.

[22] X. M. Li, H. F. Liu, X. F. Niu et al., "The use of carbon nanotubes to induce osteogenic differentiation of human 
adipose-derived MSCs in vitro and ectopic bone formationin vivo," Biomaterials, vol. 33, no. 19, pp. 4818-4827, 2012.

[23] Z. Huang, J. Tian, B. Yu, Y. Xu, and Q. Feng, "A bonelike nano-hydroxyapatite/collagen loaded injectable scaffold," Biomedical Materials, vol. 4, no. 5, Article ID 055005, 2009.

[24] Z. Huang, Q. Feng, B. Yu, and S. Li, "Biomimetic properties of an injectable chitosan/nano-hydroxyapatite/ collagen composite," Materials Science and Engineering C, vol. 31, no. 3, pp. 683-687, 2011.

[25] Z. Huang, B. Yu, Q. Feng, S. Li, Y. Chen, and L. Luo, "In situ-forming chitosan/nano-hydroxyapatite/collagen gel for the delivery of bone marrow mesenchymal stem cells," Carbohydrate Polymers, vol. 85, no. 1, pp. 261-267, 2011.

[26] Z. Huang, Y. Chen, Q. L. Feng et al., "In vivo bone regeneration with injectable chitosan/hydroxyapatite/collagen composites and mesenchymal stem cells," Frontiers of Materials Science, vol. 5, no. 3, pp. 301-310, 2011.

[27] D. H. Agemura, W. D. O’Brien, J. E. Olerud, L. E. Chun, and D. E. Eyre, "Ultrasonic propagation properties of articular cartilage at $100 \mathrm{MHz}$," Journal of the Acoustical Society of America, vol. 87, no. 4, pp. 1786-1791, 1990.

[28] D. A. Senzig, F. K. Forster, and J. E. Olerud, "Ultrasonic attenuation in articular cartilage," Journal of the Acoustical Society of America, vol. 92, no. 2, pp. 676-681, 1992.

[29] K. Hattori, Y. Takakura, Y. Morita, M. Takenaka, K. Uematsu, and K. Ikeuchi, "Can ultrasound predict histological findings in regenerated cartilage?" Rheumatology, vol. 43, no. 3, pp. 302-305, 2004.

[30] S. Kreitz, G. Dohmen, S. Hasken, T. Schmitz-Rode, P. Mela, and S. Jockenhoevel, "Nondestructive method to evaluate the collagen content of fibrin-based tissue engineered structures via ultrasound," Tissue Engineering, Part C, vol. 17, no. 10, pp. 1021-1026, 2011.

[31] K. Oe, M. Miwa, K. Nagamune et al., "Nondestructive evaluation of cell numbers in bone marrow stromal cell $/ \beta$ tricalcium phosphate composites using ultrasound," Tissue Engineering, Part C, vol. 16, no. 3, pp. 347-353, 2010.

[32] J. Wong, E. O. Gerscovich, M. S. Cronan, and J. A. Seibert, "Accuracy and precision of in vitro volumetric measurements by three-dimensional sonography," Investigative Radiology, vol. 31, no. 1, pp. 26-29, 1996.

[33] N. J. Raine-Fenning, J. S. Clewes, N. R. Kendall, A. K. Bunkheila, B. K. Campbell, and I. R. Johnson, "The interobserver reliability and validity of volume calculation from three-dimensional ultrasound datasets in the in vitro setting," Ultrasound in Obstetrics and Gynecology, vol. 21, no. 3, pp. 283-291, 2003.

[34] B. L. Partik, A. Stadler, S. Schamp et al., "3D versus 2D ultrasound: accuracy of volume measurement in human cadaver kidneys," Investigative Radiology, vol. 37, no. 9, pp. 489-495, 2002.

[35] S. M. Stieger, S. H. Bloch, O. Foreman, E. R. Wisner, K. W. Ferrara, and P. A. Dayton, "Ultrasound assessment of angiogenesis in a matrigel model in rats," Ultrasound in Medicine and Biology, vol. 32, no. 5, pp. 673-681, 2006. 

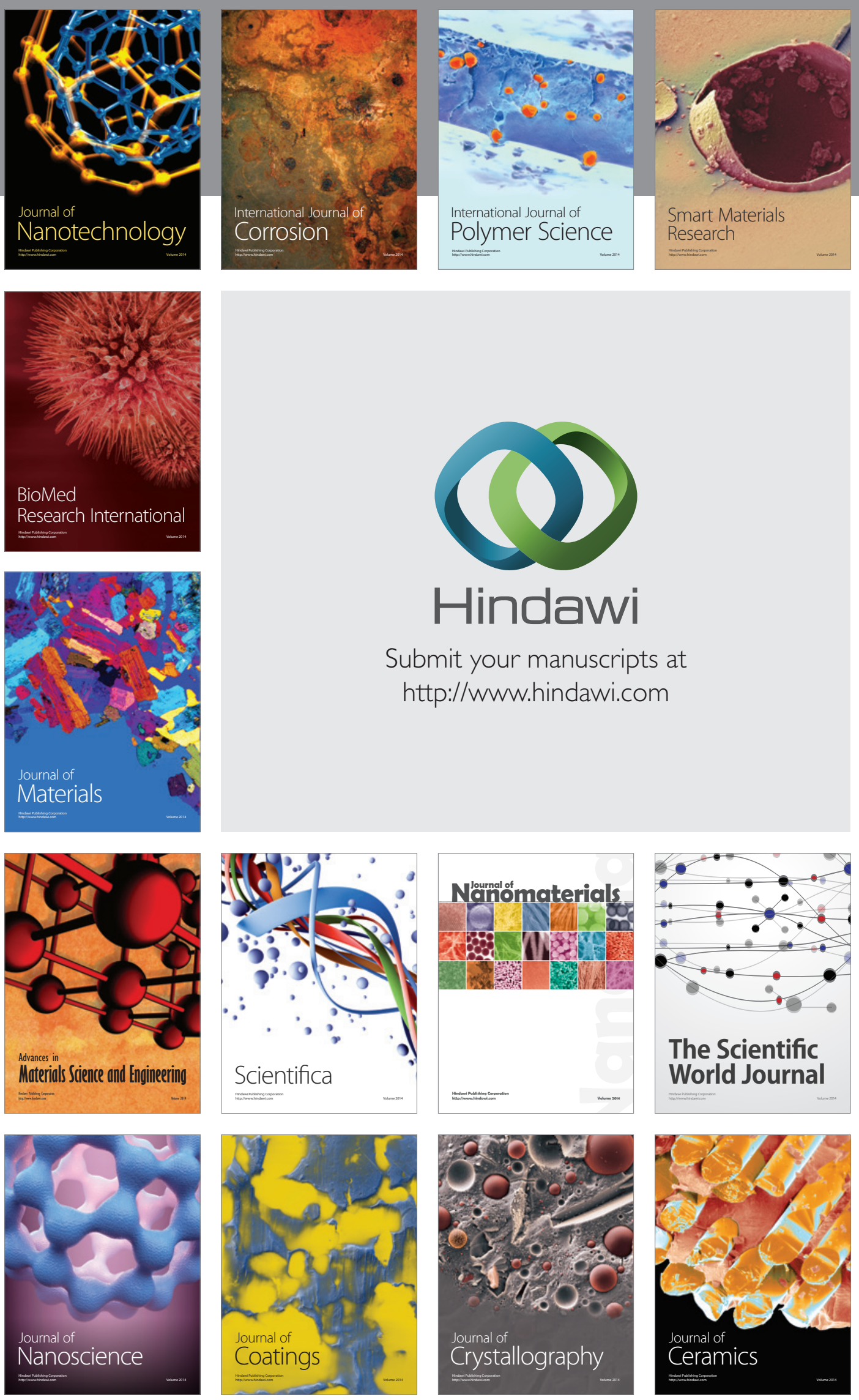

The Scientific World Journal

Submit your manuscripts at

http://www.hindawi.com

\section{World Journal}

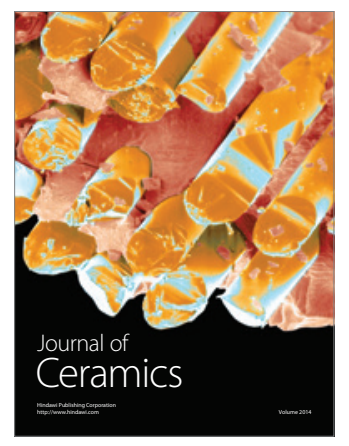

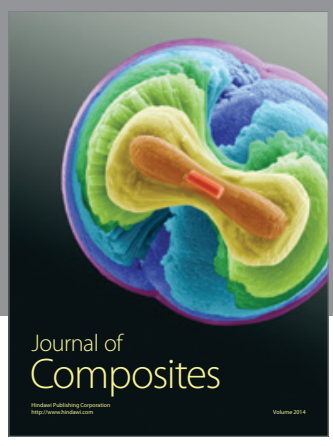
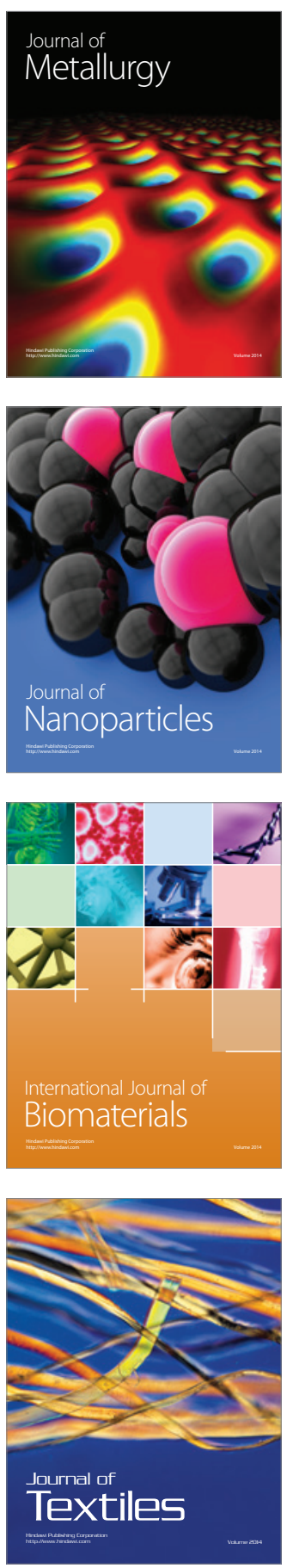